\title{
Security Constraint Optimal Power Flow (SCOPF) - A Comprehensive Survey
}

\author{
Mithun Bhaskar M, Srinivas Muthyala and Sydulu Maheswarapu \\ Dept. of Electrical Engineering \\ National Institute of Technology \\ Warangal, AP 506004, INDIA
}

\begin{abstract}
This paper reviews the existing developments in Security Constrained Optimal Power Flow (SCOPF) from 1960's to till date. Diverse schemes and approaches on Single Area/Multiarea, Algorithms, Contingency Selection, Steady and Dynamic SCOPF, Artificial Intelligence based SCOPF, Real time and SCOPF using Parallel/Distributed Processing, Economic Dispatch with Security Constraints, Decentralized SCOPF, Voltage Constrained SCOPF (VSCOPF), Incorporation of FACTS on SCOPF studies and Literatures on Applications of SCOPF etc., are appraised in a structured manner chronologically with detailed reviews on the strategies and the test systems used for the analysis are reported. A brief summary of the existing stratagems and test system data which can be retrieved are given in the conclusion for easy access of researchers.
\end{abstract}

\section{Keywords}

Optimal Power Flow, Optimization Techniques. Security Constrained Optimal Power Flow (SCOPF), Security Analysis, Flexible AC Transmission System.

\section{INTRODUCTION}

Power system throughout the world is undergoing tremendous changes and developments due to rapid Restructuring, Deregulation and Open-access policies. Greater liberalization, larger market and increasing dependency on the electricity lead to the system operators to work on limited spinning reserve and to operate on vicinities to maximize the economy compromising on the reliability and security of the system for greater profits, which lead to establishment of a monitoring authority and accurate electronic system to prevent any untoward incidents like Blackouts.

Optimal Power Flow (OPF) study plays an important role in the Energy Management System (EMS), where the whole operation of the system is supervised in each conceivable real time intervals. Optimal Power flow is the assessment of the finest settings of the control variables viz. the Active Power and Voltages of Generators, Discrete variables like Transformer taps, Continuous variables like the Shunt reactors and Capacitors and other continuous and discrete variables so as to attain a common objective such as minimization of operating cost or Social Welfare while respecting all the system limits for safe operation. This greater dependency on Electric Power has brought in the stage where the consumer depends not only on the availability of the electricity, but also looks for Reliable, Secure, Quality and Uninterrupted supply. Optimal Power flow, considered on, when system meets with a contingency viz. Generator / Transformer / Line / Load / Static or Synchronous compensator failure / Apparatus failure is termed as Security Constrained Optimal Power flow (SCOPF). The recent Blackouts lead to the importance of the system which is capable to withstand any contingencies, or to have system which can work on the specified limits when a contingency occurs, without effecting the overall operation of the system. SCOPF problem is the perfect incorporation of the contradictory doctrines of maximum economy, safer operation and augmented security.

This paper is organized into 14 Sections; First section gives an outline of OPF and SCOPF, Section II and III reviews the exhaustive segments like Steady State Security and Dynamic Security respectively, Section IV assesses the Contingency Selection strategies, Section V evaluates the Contingency Constrained OPF, Section VI deals with Security Constrained Economic Dispatch (SCED), Section VII transacts with the Security Constrained OPF (SCOPF), Section VIII analyses the Artificial Intelligence Techniques applied to SCOPF, Section IX evaluates other Algorithms and Techniques applied for Optimization in SCOPF studies, Section X censures the Voltage Security Constrained OPF (VSCOPF) approach, Section XI relate the Decentralized SCOPF approach, XII dissects the Parallel and Distributed algorithms applied to SCOPF, Section XIII reviews the methodologies of SCOPF with Flexible AC transmission System (FACTS) incorporated and Section XIV reviews the literatures on the possible application of SCOPF.

\section{STEADY STATE SECURITY}

At any given case, the security engineer monitors the power flow with induced contingencies to empower and withstand any case of overload and voltage violations. H. W. Dommel et al. [1] has sketched a detailed survey on Load flow algorithms, the first credits for Load flows unquestionably goes for J Carpentair (1962). All the considerable developments in Power Flow algorithms are listed in [2] - [3]. SCOPF studies help to overcome when any real contingency happens by rescheduling / controlling to make sure that system is within the allowed limits of operation and termed as steady state security. Earlier methods were basically of Linearized DC load flow models with many approximations [4] and used only a linearized model of only the outage system. Early 1960s has the proposal of Wells [5], and 1970s works by El-Hawary [6], Kaltenbach et al. [7] and Shen et al. [8] were the earlier works on Power system security constrained optimization, Alsac et al. [9] in early as 1973 proposed a more accurate (earlier methods were DC approximate 
methods) method to incorporate the steady state security constraints into OPF, which allowed to consider the reactive power and voltage constraints in outage cases. There, the OPF is solved using the 'Dommel-Tinney' approach and later security constraints are added to the AC-Power Flow via their penalty functions (first to introduce) and Lagrange multipliers, to obtain the optimum operating conditions which was tested on IEEE 30 bus system. But it was Monticelli et al. [10] (1987) has put forward a new blenders decomposition based method for Economic dispatch with security constraints with post-outage correction and separate the base case with contingency analysis together with generation rescheduling using Benders feasibility cuts. The method was tested on IEEE 118 bus system. Last mentioned was that of non-decomposing methods and Carpentier [11] (1973), Elacqua et al. [12] (1982), Schnyder et al. [13] (1987) and Stott et al. [14] (1978) exposed various decomposed methods involving security constraints. Dias et al. [15] (1991) claimed that by implementing the SCOPF for higher fuel cost systems, the total operating system tends to come down as the power demand on contingencies tend to come down due to lowering of voltage and natural reasons, the expensive generators are lightly loaded. He has also highlighted the effect of Under Load Tap Changing (ULTC) transformers in the normal OPF and SCOPF studies with load models implemented and modeled SCOPF solution which was tested on various $30,57,118$ and the Nova Scotia 131 bus reduced power system; with specified power demand at loads fed by tap-transformers, when specified power demand occurs at voltages obtained from the standard OPF solution and by using voltages from a standard security constrained OPF solution with and without line flow constraints in OPF and SCOPF condition. In 1996, Saavedra [16] exploited distributed processing environment with dual relaxation method and was tested on two Brazilian systems consisting of 725 buses, 1212 branches and 76 adjustable power generators; Second system of 1663 buses, 2349 branches and 99 adjustable power generators. Saavedra together with Rodrigues et al. [17] (1994) proposed asynchronous method with dual-simplex relaxation solution for the linearized SCOPF with parallel architecture processing for the preventive mode of SCOPF.

Security Constraint Optimal Power Flow becomes a biobjective problem and the optimization occurs at the best tradeoff between generation cost and the security cost. The 'Opportune Security Index', first mentioned in the Ph.D thesis of D. D Menniti (1989) and later widely used in his research on Steady State security using pattern recognition [18] and Neural Network [19] in 1991 and 1995 respectively. The very next year, Minniti together with Confroti and Sorrentino proposed Parallel Gradient Distribution (PGD) and Non-Linear Programming based OPF algorithm with (N-1) contingencies [20] with continuous security metrics and was tested on a 5 bus system.

\section{DYNAMIC SCOPF}

Ebrahim Vaahedi et al. [21] (2001) was the pioneer to include the dynamic constraints; voltage stability with the static security constraints viz., the flow and voltage profile during normal and post contingency operations. The problem has been formulated as a three level hierarchical decomposition scheme where the
Interior programming / Benders Decomposition techniques are used and tested on a North American electric utility system with 1449 buses, 2511 circuits, 778 transformers and 240 generators and on a reduced Brazilian System of 11 buses and 15 circuits and the authors has validated using Continuation Power Flow and Point of Collapse Program (PFLOW). Don Hur et al. [22] (2001) proposed a Novel algorithm in decentralized framework, using a price-based mechanism that models each region as an economic unit. Here, Linear Programming based approach is used by the authors for maximum secure simultaneous transfer capability of tie-lines.

\section{CONTINGENCY SELECTION}

Lizhi Wang [23] (2006) projected a new contingency selection technique for the SCOPF problem which proved to provide a better solution than the conventional $(\mathrm{N}-\mathrm{K})$ selection principles. A DC lossless load flow model is used and the trade-off between economy and security is achieved using a parametric utility function. A best trade-off between the Economy benefit, Infeasibility cost and Infeasibility risk are considered and Integer Programming method is used which was tested on 5 bus, 6 line system and on IEEE 30 bus system using Matlab ${ }^{\text {TM }}$ and CPLEX 9.0 platform. Y Yuan et al. [24] proposed solutions for transient stability constrained OPF with multi-contingency rather with the conventional single contingency analysis till then used. A Primal Dual Newton Interior Point method was used by authors to solve the TSOPF problem on 3 machines, 9 bus systems; IEEJ WEST 10 and IEEJ WEST 30 systems and implemented using FORTRAN language. Francois Bouffard et al. [25] (2005) proposed a model to Identify, Analyze and Validate a set of contingencies from the complete set of contingencies by norms of the Lagrange multiplier vectors of Post-contingency analysis. Two cases, viz. the Deterministic case and the Stochastic SCOPF problem have been analyzed here. In the former, the umbrella contingencies are identified as the contingency which yield the same market-clearing solution and in the latter case, the identification is done such that the sensitivity of the optimum solution to the neglected contingencies is smaller to a prespecified threshold. In addition, authors have attempted to validate "super umbrella" contingency, which are nothing but, umbrella contingencies which remain as umbrella contingencies irrespective of the system parameters. A DC-OPF analysis was used for Numerical analysis of deterministic SCOPF problem as the load varies and the effect of ranking and other cut-off rules of stochastic SCOPF is demonstrated and is tested on a Three-bus, three-line, three-generator system.

Florin Capitanescu [26] (2007) introduced two novel contingency filtering techniques based on comparison of intermediate solutions of Preventive Security Constrained OPF (PSC-OPF) in post contingency analysis. Authors have compared the proposed method with classical methods like severity indexbased (SI) filtering schemes and with direct PSCOPF method. Two techniques viz., Individually Non-dominated Contingency (INDC) Technique and Non-dominated Contingency Group (NDCG) Technique; both based on the concept of 'constraint violation domination'. Both had the advantage that it is free from any parameter tuning (weight matrices and thresholds). The 
former technique is found to keep only the non-dominated contingencies, which can obtain a solution to PSOPF, as of all contingencies are present and discarding all other ones; the later technique selects the contingency for each constraint, which creates maximum violation. The Interior-Point Method (IPM) is used in the base algorithm. The algorithms were tested on a modified Nordic32 system (60 bus system) and on standard IEEE 118 test bus system. Authors demonstrate that the proposed methods are more robust and accelerate the sequential solution of PSOPF than any other classical methods.

\section{CONTINGENCY CONSTRAINED OPF}

Ramesh et al. [27] (1997) put forward a decomposed form of Contingency Constrained Optimal Power Flow (CCOPF) using Fuzzy Logic where, the minimization of both the base case (precontingency) operating cost and of the post-contingency correction times which are conflicting, were accepted as fuzzy goals. Devaraj et al. [28] (2005) demonstrated a new Real Coded Genetic Algorithm (RCGA) centered approach for OPF for improving the security goals of line overload by generation redispatching and by adjustment of phase-shifting transformers, which are installed based on the Severity Index (SI). This algorithm has overcome the traditional GA snags of solution being depended on the number of bits of the variables and the cumbersome procedure of converting the real time variables into binary strings. The variables are modeled in natural form and operating the cross-over and mutation operators directly with integer and floating-point genetic algorithm. The authors has adopted the IEEE 30 and IEEE 118 bus systems for implementation and three cases viz, for obtaining the optimalcontrol variables in the IEEE 30-bus system; to alleviate overloads under line outage by generator rescheduling and phaseshifting transformers; the third case, proposed algorithm was used to alleviate line overload in the IEEE 118-bus system. Lopez-Lezama [29] (2006) offered a new coupled post contingency OPF with reliability criteria added as an additional linear constraint. The algorithm was tested on a Colombian market. The actual nodal prices and marginal price of a blackoutrisk are also calculated. The mathematical modeling of that paper for SCOPF was adopted from Thorp et al. (2001) by using of coupled post contingency Optimal Power Flows and a unique system of islands are build, which are nothing but the base case system and the system after contingency. Load of various types like Dispatchable, Curtailable and Sheddable are found to be included. Objective function modeling consisting of additional variable; the difference of expected relative load shedding and average of all the expected relative load shedding, together with probabilities of contingencies are included and MATPOWER has been used for optimization part by authors.

\section{ECONOMIC DISPATCH WITH SECURITY CONSTRAINTS}

Mohamed Aganagic et al. [30] (1997) demonstrated a two level decomposition algorithm using nonlinear version of the 'Dantzig-Wolfe' decomposition based Security constrained Economic dispatch (SCED) using nonlinear unit cost functions. A detailed representation of the reserve curves were given and were tested on three custom test cases with a total load of 3595MW. The proposed algorithm consists two phases; first phase obtains a primal feasible solution by minimizing the sum of infeasibilities, whereas in second phase the generation cost is minimized and is solved using a revised simplex method. Yan et al. [31] (1997) unraveled the Security Constrained Economic Dispatch (SCED) using successive linear Programming/Predictor-Corrector Interior Point method. Efforts have been made for the adjustment of Barrier Parameter and for determination of initial points. The proposed algorithm is compared with the results obtained using primal-dual interior point method. The algorithm is not applied directly; instead, the successive linear programming is applied to exploit the computational gain achieved on not having to calculate the second-order derivatives of Hessian matrix at each iteration. The proposed algorithm was tested on 236, 354, 708, 1062, 2124 bus systems, which are obtained by interconnecting standard IEEE 118 bus systems in many ways. Authors concluded by using feasibility condition on fast reducing duality gap, by customizing initial points by adopting relatively small threshold and by balancing its primal and dual values, the number of iterations can be reduced even up to $50 \%$ and the time savings are found to increase with larger size systems. Luis Vargas et al. [32] (1993) published an in-depth tutorial on Interior point method and demonstrated the application of IP (Dual Affine version) on SCED problem. Luis deliberated on the superiority of the IP over the simplex method and a demonstrated a practical method to avoid the oscillatory behavior in the iteration process of IP. Fast Decoupled Load Flow, Generalized Generation Distribution Factors (GGDF) and generation power Incremental Transmission Losses Factor (ITLF) concepts are used in sub problems of the proposed algorithm and to increase the computational speed, 'Pre-conditioned Conjugate Gradient' (PCG) technique is used instead of the direct method based on Cholesky factorization, to prevent ill-conditioning and added time consumption. The algorithm was implemented and tested on IEEE 30 and 118 bus systems and is compared with simplex code (MINOS). Rabih Jabr et al. [33] (2000) put forward a new simplified homogeneous and self-dual (SHSD) linear programming (LP) interior point algorithm for SCED and did the analysis not only for the conventional (N-1) criteria but also for the $(\mathrm{N}-2)$ contingencies. The analysis has been compared with predictorcorrector interior point algorithm as proposed by Yan [31]. The cost curves in this paper are considered as convex and piecewiselinear and expressed in a separate programming which is tested on an IEEE 24 bus test system and on a practical 175 bus network. Yong Fu et al. [34] (2006) made an attempt on modeling a Security constrained Unit Commitment (SCUC) model with preventive/corrective approach contingencies (controllable and uncontrollable) over an $24 \mathrm{hr}$ time schedule, AC-SCOPF, Load shedding and Unit commitment is considered, wherever the security constraints are not met in the recalculation of Unit commitment. The Authors have exploited Augmented Lagrangian relaxation, Dynamic programming and Benders Decomposition for solving the SCOPF/SCUC/UC. Load Shedding is resorted for unfeasible problem arising out of contingencies to act as Virtual generators based on decremental bids. Authors have adopted 6 bus, IEEE 118 bus and 1168 bus (169 generators, 1168 buses, 1474 branches, and 568 load sides) 
test systems for implementation and the AC results are depicted. It's concluded that the implementation time increases linearly with size of the problem. Kyoung Shin Kim [35] et al. (2006) approached the SCED with Interior Point method by including the power flow constraints. An novel algorithm is presented to linearize the SCED problem based relations among generator outputs, active power flows, loads, losses etc. and is solved using Linear programming. The concepts of Incremental Transmission Loss Factor (ITLF) and Generalized Generation Distribution Factor (GGDF) concepts are used in the algorithm which is later optimized using Primal Interior Point Method (PIPM). Authors has compared the results obtained by applying this algorithm to IEEE 6-bus and 30-bus systems and comparing it with Simplex Programming and its found that this algorithm offers more computational speed and takes lesser iterations. Florin Capitanescu et al. [36] (2008) proposed new techniques to solve the Corrective Security Constrained Optimal Power Flow (CSCOPF) consisting of CSOPF, Steady State Security Analysis (SSSA), a contingency filtering and an OPF variant to check post contingency corrective analysis. Severity-Index-Based Contingency Ranking Approach, Non-dominated Contingency (NDC) Approaches are used by authors in the CF category. Other variant of new Iterative CSCOPF approach like Infeasible postcontingency optimal power flow, without filtering and Severity based approaches are also demonstrated in the paper. Authors have analyzed the proposed method with classical direct approach and Benders decomposition techniques and are tested on modified 60 bus (Nordic32), IEEE 118 and 1203 bus (FrenchRTE) systems and the algorithm is found to be more robust and faster than direct approach, Benders decomposition technique and severity index (SI) based approaches.

\section{SECURITY CONSTRAINED OPF}

Harshan et al. [37] exposed notable works in speeding up the SCOPF analysis to make them competent for the online analysis using with a new fast Cyclic Contingency Screening model (CCS) of security analysis by accepting the results of a security analysis carried out at time ' $t_{\mathrm{k}}$ ' for drawing the data to be used in a security analysis at a time ' $t_{k}+A t^{\prime}$ to reduce the computational burden which in turn increases the speed of the entire analysis. Authors used local perturbation effect and Concentric Relaxation Method and double stage pre-filters to separate non-critical cases on updating the database. This method was tested on National French $225-400 \mathrm{kV}$ grid containing 462 nodes and 855 branches with 96 real states for a $24 \mathrm{hr}$ period on 15 minute steps. Fabrice Zaoui et al. [38] (2006) proposed a new direct method for the simultaneous optimization of AC-OPF base case and with $(\mathrm{N}-\mathrm{K})$ contingencies rather having sub problem approach which has been commonly found, using Primal Dual Interior Point method (IPM). The contingency analysis is run before the optimization process to select only the critical contingencies as size of the optimization problem increases linearly with the number of considered contingencies. In this approach, IPM converts the inequality constraints to the equality ones with addition of two positive slack variables and the equality constrained problem is converted to an unconstrained problem by using Lagrangian function together with a 'Fiacco-McCormick' approach for barrier update (also called monotone strategy). The algorithm is tested on a small 3-bus network, medium system of 95 buses, 105 branches with 19 load tap changing transformers, 22 generating units and 21 shunt compensation devices (Corsica Island) \& on a large system consisting of 1207 buses, 1821 branches, 185 generating units and 2 shunt compensation devices (French Continental Network). Oñate Yumbla et al. [39] (2008) proposed a Particle Swarm Optimization with Reconstruction Operators (PSO-RO) based solution for SCOPF problem, where the constraints are handled using reconstruction operators, instead of penalizing the objective function. Authors have formulated the OPF with (N-1) criterion, where the Pre/Post optimal contingency points are obtained, together while considering the constraints in generating units' limits, minimum and maximum up and down-time, slope-down and slope-up, and coupling constraints in the pre- and the post-contingency states. Authors claim that by PSO-Reconstruction Operator approach, there is an increase in the search area/particles in the space. Performance Index based Contingency ranking system is used together with NRLF and is tested on two systems; viz. 39 buses, 46 branches, ten generators (New England System) with a total load of 1000MW and another one consisting of 26 buses, 46 branches, six generators, seven transformers, and nine shunt capacitors (adopted from Hadi Sadat) with Six active power generations, Seven transformers-tap setting, and Nine varinjection values with a total load is $1263 \mathrm{MW}$ and 22 control variables.

\section{ARTIFICIAL INTELLIGENCE TECHNIQUES}

Literatures speak that the main constriction was that the problem tend to settle in a global optimum as security constraints are difficult to be included in the line security constraints into fitness function. L L Lai et al. [40] (1997) proposed an Binary Coded Improved Genetic Algorithm approach for the Normal and contingent condition of the system and two cases has been compared on IEEE 30 bus system with a simulated circuit outage. Somasundaram et al. [41] put forward a Evolutionary programming based solution for the SCOPF problem and claimed to be a better and robust technique as EP uses only the objective function information and not the first and second derivatives of it or constraints and is independent of the nature of the search space such as smoothness, convexity or uni-modality and is tested on a IEEE30 bus system. Zwe-Lee Gaing et al. [42] (2006) suggested a Real coded Mixed Integer Genetic Algorithm based approach to the SCOPF problem. There, real coding is exploited instead of the conventional binary coding with a two arithmetic crossover and mutation schemes are proposed. Authors used uniform crossover, FDLF method and only one critical contingency is selected among all for the contingency analysis and the results provide a comparison with that of evolutionary programming on same system. The proposed SCOPF not only considers the generation cost, but also transmission security, transmission loss, bus voltage profile, value-point loading and is tested on custom 26 bus (46 transmission lines, load demand of 1263 MW system) \& IEEE 57 bus system. 


\section{OTHER TECHNIQUES TO SCOPF}

It was in 1997, Scott et al. [43] reviewed some exceptional works on their 'Invited paper' on Power System Security Optimization Techniques, which revealed the future scope of online contingency analysis and pointed out areas of difficulty that constitute them and challenges for successful practical online implementations which are applied for the security analysis of Power System in the future. An in-depth review of security concepts and terminology, Security Assessment, Optimization techniques; Linear and Non-linear, a thorough ideas on modeling of Contingency Analysis, Direct \& Indirect Contingency Selection methods, Active and Reactive power Contingency Screening, Security Constrained Optimal Scheduling; Contingency constrained OPF with security level 1 and level 2; online security analysis and its found to be must read literature for any researchers on Power System Security. Momoh et al. [44] offered a Quadratic Programming and New Non-Linear Convex Network Flow Programming (NLCNFP) model, which considers the tie-line security and transfer constraints together with buying and selling contracts, has been implemented on a four area IEEE 30 bus system. Karim Karoui [45] (2008) highlighted the use of Interior Point programing (Interior-point Direct, Interior-point CG, Active set algorithms) for SCOPF using KNITRO (Integrated Power System Optimizer) software, which offers preventive and corrective strategy, the discrete variables modeling, the modeling of units capability curves, the modeling of the primary active power-frequency control, modeling of discrete variables, Transformer taps discretization with shunt variables discretization and authors has demonstrated the use of the same in evaluating the Total Transfer Capability. It's found that KNITRO models the problem into barrier method where, nonlinear objective function is replaced to a set of barrier sub-problems controlled by a barrier parameter. The algorithm is demonstrated on a 2351 bus, 4587 lines European system. Anibal et al. [46] proposed a Predictor-Corrector Interior Point algorithm for SCOPF problem with Branch Outages, Generator Outages and Multiple equipment congestion together with the objective of minimization of transmission loss. A scalar weight method is used for integrating the objectives together. Line Outage Distribution Factors (LODFs) and Generalized Generation Distribution Factors (GGDFs) are used in the contingency analysis consisting of 157 security constraints. Authors have validated the algorithm on a Brazilian power system consisting of 3535 bus and 4238 branches for a tolerance of 0.01 .

\section{VOLTAGE SCOPF}

Claudio Canizares et al. [47] (2001) projected and compared two OPF techniques incorporating Voltage Security, both multiobjective optimizations, minimizing the generation cost, transmission losses and improving the voltage security. The minimum voltage collapse point constraint is added together with the singular value and its derivatives at each iteration using the Hessian of the power flow equations which is solved using the Han-Powell procedure; Nonlinear Primal-Dual PredictorCorrector Interior Point method and is tested on a modified IEEE 118 bus system. Devaraj et al. [48] (2007) proposed a new Improved Genetic Algorithm for the voltage security constrained
OPF which used the natural form of the variables (Real coded GA) with floating point integers based crossover and mutation probability. Generator power output, Generator voltage magnitude, Transformer Taps and Reactive power of the capacitor banks are selected as control variables and the voltage stability is analyzed using the Maximum L-index value of load buses. In the proposed genetic algorithm the continuous variables are modeled as floating point numbers and discrete variables as integers. The method has been tested on a standard IEEE 30 bus system. Worawat Nakawiro et al. [49] (2009) proposed a novel GA-ANN method for network loss minimization and for the reactive power dispatch where the ANN are trained offline to substitute for OPF online. The k-mean clustering method is used to select the input for the ANN, Line Indicator (L) is used to analyze the security margin and GA is used for optimizing the complete problem. For the offline learning, a database encompassing realistic operating conditions, in terms of random load, generation mix and outages is simulated on 6000 operating points which were selected for ANN training using Back propagation method (Lavenberg-Marquart optimization adopted). The proposed algorithm was tested on a Standard IEEE 30 bus system and it's found to be 5 times faster than other conventional methods.

\section{DECENTRALIZED OPF}

Biskas et al. [50] (2005) presented a decentralized solution for large interconnected system by decomposing multi-area system SCOPF problem into smaller individual SCOPF problem. Later, the sub problems are combined using a pricing mechanism, which are the electricity exchange prices of the neighboring areas, until they converge all smaller sub problems. The advantage of this method is found to be reduced effect of line outages. Unit outages outside the sub problem area are ignored. Authors have implemented this algorithm for an IEEE 3 area RTS-96 and Balkan Power system consisting of 310 buses, 77 units, 485 internal lines and 5 tie-lines. Don Hur et al. [51] presented a new parallel decentralized solution for SCOPF problem using Linear Programming using Line Outage Distribution Factor and was implemented on the Korean Power System consisting of four regions and each region connected directly by the major eight $345 \mathrm{kV}$ transmission lines and seventeen $154 \mathrm{kV}$ transmission lines, which are considered as tie lines. The intraregional SCOPF is solved using conventional Linear Programming (LP) approach by the authors.

\section{PARALLEL PROCESSING BASED OPF}

Wei Qiu [52] (2005) proposed a new parallel processed (16 Pentium $1 \mathrm{GHz}$ Processors used in paper) solution for SCOPF using Nonlinear Interior Point Method (Primal-dual interior point). Authors have used multiple set of distributed processors for independent and parallel computing of contingency states. The 'Blocked Diagonal Bordered' (BDB) structure of the linear equations is exploited for assigning each processor an independent block for parallel processing. Authors have used 'Generalized Minimal Residual' (GMRES) method solutions have been used for faster convergence. Its claimed that the proposed algorithm gives 12 times faster solution for 
decomposed SCOPF problem, based on the tests implemented on one with 57 buses, 80 branches and 9 contingencies, another with 3493 buses, 6689 branches and 79 contingencies and is compared with MatPower software. Carmen et al. [53] (2007) was the first to demonstrate a SCOPF in real time using distributed processing environment for an Energy Management System (EMS - supposed as National System Operation Center). Authors used 'Message Passing Interface' system (MPI) for parallel processing, Interior Programming for the optimization and Benders Decomposition technique for solving SCOPF. The authors have best explained the previous developments like 'Parallel Virtual Machine' (VPM) and FLUPOT - Interior programming technique from the previous literatures. The conventional method of base case and contingency induced subcase is followed in this paper too. During unfeasible contingency cases, penalties are levied to represent the quantity of variation. In the proposed algorithm, reading of input and base case analysis is done simultaneously by all processors, whereas reorganization of the Benders-Cut data structure are done by using distributed computing, where each slave processor solves its own contingency list and produces its own partial benders-cut, which are sent to the Master processor, which in turn reorganizes all collected data from Slave processors into a new benders-cut structure. Later, it's found that SAGE - 'Sistema Aberto de Gerenciamento de Energia' system integrates the collected distributed SCOPF data into SCADA/EMS. 16 dual-processed nodes cluster of computers are put on work for a large real time Brazilian system consisting of 3073 bus, 4547 branches, 963 active and reactive controls with 700 contingencies, 57,947 MW and 16,007 Mvar total load with a generation of 60,698 MW and 19,112 Mvar, losses are 2,751 MW and 3,105 Mvar. The authors claim to have achieved an efficiency increase of $92 \%$ and processing speed decreased to $111.16 \%$ and much economic profits as number of additional hardware is require to integrate this system to SCADA/EMS is lesser than other conventional approaches. Saavedra et al. [17] (1994) offered parallel architecture processing for the preventive mode of SCOPF using Asynchronous Dual-Simplex method. Don Hur et al. [22] (2001) approached parallel SCOPF problem in a decentralized framework, using a price-based mechanism that models each region as an economic unit. Here an approach based on Linear Programming is used and post-contingency constraints are linearized to append to the base case in every iteration. There, OPF problems is solved for maximum secure simultaneous transfer capability of each tie-line between adjacent regions by taking only the Security Constraints imposed on the tie-lines into account and by using the Line Outage Distribution Factors (LDF). In the next stage the maximum secure transfer capability of tie-lines are solved in such a way that the system stays in limit even when a generator is removed. GAMS 2.25 software is used for the parallel computing using the in-built MINOS5 and CONOPT solvers on a test system of two area Korean Power System and the maximum allowable flow on all tie-lines is tabulated in the conclusive statement of authors.

\section{FACTS INCORPORATED SCOPF}

Carsten Lehmköster [54] (2002) validated the modeling of FACTS and contingencies, their analysis and optimization with for different objective functions and constraints using Sequential Quadratic Programming / Gradient method. The system has been well demonstrated and tested on an IEEE 118 bus system. Alberto Berizzi et al. [55] (2005) proposed a compact and reduced SCOPF formulation with FACTS devices as control variable and used 'Han-Powell' algorithm to solve the OPF. The approach used a new penalty related to control variables of FACTS with improved sensitivity calculation; reactive Jacobean and the voltage displacement approach to find a global solution for the entire system with a single quadratic equality constraint representing the real power balance of the total production, load, losses, and a reduced set of constraints on the current flows. The paper modeled two mono-dimensional; Series Power Flow Control (SPFC), Thyristor-Controlled Phase Angle Regulator (TCPAR) and one bi-dimensional; Unified Power Flow Controller (UPFC) FACTS devices applied to the most heavily loaded lines and (N-1) security analysis (29 contingencies were used by authors) is performed on a the CIGRE 63-bus system of 2080 MW total load and an Italian EHV network of 1414 buses, more than 300 generators and with a total load of about 34000 MW. The authors concluded that the proposed algorithm is very fast for mono-dimensional devices and is found to be time consuming for bi-dimensional FACTS devices. Glanzmann et al. [56] (2006) developed a current injection method by which the power flow in any line during an outage can be calculated using linear equations. The current injection method was tossed by Bacher et al. (1986) and Schnyder et al. (1988), which has the advantage of not having to go through the cumbersome rebuilding of Power Flow iteration in case of any contingency and is implemented by the authors in this paper. An excellent illustration of the current injection method is given in the paper and authors have introduced Additional Bus Injection Currents in the proposed method to all other buses other than the load and generator bus. Total susceptance or reactance model concepts are used for the inclusion of the FACTS devices (SVC \& TCSC) in the system. Only critical contingencies are considered by the authors in the inclusion of $(\mathrm{N}-1)$ contingencies in the later stage with an IEEE 57 Bus grid with additional generator at bus 30 is used as a test system. Narmatha Banu et al. [57] proposed a new enhanced Genetic Algorithm based solution for SCOPF with FACTS (TCSC) incorporated and the optimal location of the FACTS are found using Overload Index Factor. The authors have predetermined the possible sites for the installation of the TCSC during various contingencies, by selecting the most overloaded lines and by selecting the best four lines beside it to place TCSC and there after the Genetic Algorithm is used to find the optimal values. The results (Fuel Cost, Generation) has been compared with Evolutionary Programming and with the method opted by Alsca (1973- using penalty function) and it's found that authors' proposed method offers the best solution and security for an IEEE 30 bus system. Prasanna et al. [58] proposed a new combinational algorithm for multi-area SCOPF incorporating Fuzzy Logic in Evolutionary Programming and Tabu Search and termed it as Fuzzy Muted Evolutionary Programming (FMEP) and Fuzzy Guided Tabu Search (FGTS). The proposed algorithm has been tested on single area IEEE 30 bus system and 
interconnected two area systems formed by joining two IEEE 30 bus system thorough a tie-line of scheduled interchange. Fuzzy logic is incorporated to expedite the convergence. The search range factor and scaling factor are improved using Fuzzy in the proposed algorithm to make it adaptive. Three single line outages are considered by the authors in the paper and a conclusive statement on the other possible applications of this algorithm is well given. Hug-Glanzmann et al. [59] (2009) proposed a sensitivity based method to find the reduced area optimization with FACTS device incorporating (N-1) security criteria. Current Injection method (voltage/bus currents is given as a function of the decision variables in the base case) is adopted to determine the system state in case of contingency, complete Load Flow need not to be run. Reduced Area method is found to be an attractive option as the influence of FACTS are limited to certain area and the complete area need to be considered for optimization in SCOPF analysis. Sensitivity Analysis is used to find the area of influence and the simulations are validated using 'fmincon' in MATLAB TOMLAB. The proposed method and is tested on a modified IEEE 57 bus system (additional generator at bus 30 ) and the results are found to be promising.

\section{MISCELLANEOUS APPLICATIONS}

Florin Capitanescu et al. [60] (2006) narrated much of the importance of SCOPF with main stress on three factors; Generation cost minimization, Cost of removing congestion and Maximum power transfer (Loadability margin) computation. Newer developments like introduction of surrogate power flow limits have been stated. Modeling of SCOPF in 'preventive approach' (PSCOPF) and 'corrective approach' (CSCOPF) has been explained and to prevent unrealistic variations of control variables under the effect of contingency, "coupling" constraints between the base case and post-contingency values of control variables are included in this approach. An interior point (Primal Dual Interior Point) approach is narrated and the inequality constraints are eliminated by adding them to the objective function as logarithmic barrier controlled by a scalar "barrier parameter", which gradually comes down to zero with iterations. The system adopted for testing is a NORDIC32; 60 bus system, where, above mentioned three factors are analyzed for cases like OPF with no security constraints; PSCOPF; and CSCOPF. The power of the proposed algorithm to withstand congestion is tested by overloading the test case up to $147 \%$. The authors have discussed in deep, the critical issues connected to SCOPF like effect and tediousness in analyzing the large number of contingencies in a real system, contingencies filtering techniques and about identification of umbrella contingencies, Parallel processing techniques etc. Jayashree et al. [61] (2008) explained the newer application of SCOPF in deregulated market by developing a coupled unified model of multi-transaction based
SCOPF with Available Transfer Capability (ATC) and Congestion Management. Authors have used Successive Linear Programming and Fast Decoupled Power Flow method for linearized AC-PF analysis and Multi-transaction framework of Shu \& George (2002) was adopted for analysis. The algorithm enforces the line flow limits and two cases viz., with fixed fractions assigned to participants and another one with flexible fractions in multi-transaction are evaluated on a three area IEEE 24 bus reliability test system and 119 bus Indian Utility system consisting of 25 generators, 168 transmission lines, 26 transformers, 3 shunt reactors and shunt capacitors. It's found that there is an improvement of $8.74 \%$ and $4.26 \%$ without and with security constraints respectively. Sarbjeet Kaur Bath et al. [62] (2007) proposed a Multi-objective Security Constrained OPD solution by minimizing various objectives like Operating cost, Emission together with variance of active power generation and reactive power generation. A stochastic approach is followed here with Variations in system parameters and variables are modeled mathematically using their variance and covariance. Fuzzy Logic technique has been exploited to model various objectives and membership functions are modeled for Security of transmission lines with respect to expected active power flow. Again, the 'G.E.P. Box's' Evolutionary search technique is made use for searching the 'weight factor' which are used for trade-off in integrating conflicting multiple objectives and NR method is used for solving Lagrange's function in each weight factor combination. In this paper, a hypercube search pattern is followed by EP, where weight combinations are simulated at corner points of multi-dimensional hypercube, which forms around an initial search point, which constantly moves to a better 'fit' place. Security of the transmission lines considered in the proposed algorithm and the authors have made a novel attempt by converting the stochastic models into deterministic models by cogitating their expected values with basic statistical principles. Robert Thomas et al. [63] proposed an advanced SCOPF algorithm in a co-optimization framework in which contingencies, ancillary services, and network constraints are taken care of. The proposed method is a full AC non-linear OPF method with (N-1) contingencies of both dynamic and static in nature. Proposed method also offers procuring an adequate and locational relevant supply of real and reactive power to meet critical contingencies, sets day-ahead contract prices for reactive power and for reserves and re-dispatch of load and supply for next day. There was no demonstration of the proposed "super $O P F^{\prime \prime}$ to any test systems, but a wider literature survey was conveyed. 
Table 1. Summary of various methods/algorithms and test systems used in literatures

\begin{tabular}{|c|c|c|}
\hline Area & Test Systems Used & Methods/Algorithms/Techniques most found \\
\hline \multirow[t]{2}{*}{ SCOPF } & $\begin{array}{l}\text { Brazilian system ( } 3535 \text { bus) } \\
\text { Brazilian System ( } 11 \text { bus) } \\
\text { Colombian System ( } 20 \text { bus) } \\
\text { Corsica Island ( } 95 \text { bus) } \\
\text { European system ( } 2351 \text { bus) } \\
\text { French Continental ( } 1207 \text { bus) } \\
\text { French-RTE } 1203 \text { bus system } \\
\text { IEEE } 118 \text { bus system } \\
\text { IEEE } 14 \text { bus system } \\
\text { IEEE } 24 \text { bus reliability test sys } \\
\text { IEEE } 30 \text { bus system } \\
\text { IEEE } 5 \text { bus system } \\
\text { IEEE } 57 \text { bus system } \\
\text { IEEE } 57 \text { bus system } \\
\text { IEEJ WEST } 10 \text { \& IEEJ WEST } 30\end{array}$ & $\begin{array}{l}\text { Artificial Neural Network } \\
\text { Asynchronous programming } \\
\text { Benders decomposition } \\
\text { Benders Decomposition technique with feasibility cuts } \\
\text { Binary Coded Genetic Algorithms \& it's variants } \\
\text { Dual relaxation method } \\
\text { Dual-simplex relaxation method } \\
\text { Evolutionary Programming } \\
\text { Fast cyclic contingency screening model } \\
\text { Fuzzy Logic } \\
\text { Interior Programming } \\
\text { Mixed Integer Genetic Algorithm } \\
\text { Mixed Integer Programming }\end{array}$ \\
\hline & $\begin{array}{l}\text { Indian Utility system ( } 119 \text { bus) } \\
\text { Korean System } \\
\text { Korean System (Two area) } \\
1663 \text { bus, } 24 \text { bus, } 3 \text { bus \& } 725 \text { bus } \\
26 \text { bus system (Hadi Sadat) } \\
\text { National French } 225-400 \text { kV sys } \\
\text { New England System ( } 39 \text { bus) } \\
\text { NORDIC32 ( } 60 \text { bus system) } \\
\text { Nordic32 } 60 \text { bus system } \\
\text { N American system ( } 1449 \text { buses) } \\
\text { Nova Scotia } 131 \text { bus system }\end{array}$ & $\begin{array}{l}\text { Nonlinear convex network flow programming (NLCNFP) } \\
\text { Linear Programming } \\
\text { Predictor-Corrector Interior Point } \\
\text { Primal Dual Interior Point } \\
\text { Primal Dual Newton Interior Point method } \\
\text { PSO with reconstruction operators } \\
\text { Quadratic Programming } \\
\text { Real Coded Genetic Algorithm \& It's variants } \\
\text { Successive Linear Programming } \\
\text { Tabu Search }\end{array}$ \\
\hline $\begin{array}{l}\text { Decentralized } \\
\text { SCOPF }\end{array}$ & $\begin{array}{l}\text { Balkan Power system } \\
\text { IEEE } 3 \text { area RTS-96 } \\
\text { Korean } 4 \text { area system }\end{array}$ & $\begin{array}{l}\text { Linear Programming } \\
\text { Quadratic Programming }\end{array}$ \\
\hline $\begin{array}{l}\text { Parallel Processing } \\
\text { SCOPF Algorithms }\end{array}$ & $\begin{array}{l}3493 \text { bus, } 57 \text { bus system } \\
\text { Brazilian system ( } 3535 \text { bus) } \\
\text { IEEE } 5 \text { bus system }\end{array}$ & $\begin{array}{l}\text { Linear Programming } \\
\text { Parallel Gradient Distribution } \\
\text { Primal Dual Interior Point }\end{array}$ \\
\hline SCED & $\begin{array}{l}\text { IEEE } 118 \text { bus system } \\
\text { IEEE } 24 \text { bus system } \\
\text { IEEE } 30 \text { bus system } \\
\text { IEEE } 6 \text { bus system } \\
1168 \text { bus system } \\
236,354,708,1062,2124 \text { bus systems } \\
\text { Practical } 175 \text { bus system }\end{array}$ & $\begin{array}{l}\text { Augmented Lagrangian relaxation method } \\
\text { Benders Decomposition } \\
\text { Dynamic programming } \\
\text { Interior Point (Dual Affine version) Preconditioned Conjugate } \\
\text { Gradient method } \\
\text { Linear Programming } \\
\text { NL version of the Dantzig-Wolfe decomposition } \\
\text { Revised simplex method }\end{array}$ \\
\hline
\end{tabular}




\begin{tabular}{|c|l|l|}
\hline \hline \multirow{5}{*}{} & & $\begin{array}{l}\text { Predictor-corrector interior point method } \\
\text { Primal Dual Interior Point Method } \\
\text { Primal-dual interior point method } \\
\text { simplified homogeneous and self-dual (SHSD) -linear programming } \\
\text { (LP) interior point algorithm } \\
\text { Successive Linear Programming }\end{array}$ \\
& & $\begin{array}{l}\text { Current Injection Method } \\
\text { Gradient based optimization } \\
\text { SCOPF - FACTS }\end{array}$ \\
& $\begin{array}{l}\text { CIGRE 63-bus system } \\
\text { IEEE 118 bus system } \\
\text { IEEE 57 bus system } \\
\text { Italian EHV network (1414 bus) }\end{array}$ & Sequential quadratic programming \\
\hline \hline
\end{tabular}

\section{CONCLUSION}

This paper has tried to review all the available publications in the area of Security Constrained Optimal Power Flow from early 60's to 2009. Much deeper insight to the allied areas like Contingency selection, Parallel Processing and Distributed OPF, Voltage Security Constrained OPF, Preventive SCOPF and Corrective SCOPF, Earlier developments on Security Constrained Economic Dispatch (SCED) and SCOPF with FACTS incorporated with deep review of algorithms and Stratagems of the literatures are provided and are concluded with a review on various applications of SCOPF. A summary of the available techniques, algorithms and various test system data's which can be retrieved from literatures are given in Table. 1 for the easy rescue of researchers.

\section{REFERENCES}

[1] H. W. Dommel and W. F. Tinney, " Optimal load flow solutions," IEEE Trans. on Power Apparatus and Systems, Vol. PAS-87, pp. 1866-1876, Oct. 1968

[2] J. Carpentier, "Optimal Power Flows: Uses, methods and developments", IFAC Symposium on planning and operation of electric energy systems. Rio de Janeiro, July 1985 (Invited survey paper)

[3] M. Huneault and F. D. Galiana, "A Survey of the Optimal Power Flow Literature," IEEE Trans. Power Systems, vol. 6, pp. 762-770, May 1991

[4] N. M. Peterson, W. F. Tinney \& D. W. Bree, "Iterative linear power flow solution for fast approximate outage studies", IEEE Trans. on Power Apparatus and Systems, Vol. PAS-91, pp. 2048-2053, September/ October, 1972

[5] Wells, D.W., "Method for Economic Secure Loading of a Power System", IEE Proceedings, Vol. 115, No. 8, pp. 11901194, Aug. 1968

[6] El-Hawary, M.E. and Dias, L.G., "Bus Sensitivity to Model Parameters in Load-Flow Studies", IEE Proceedings, Vol. 134, Part C, No.4, pp. 302-305, July 1987.

[7] Kaltenbach, J.C. and Hajdu, L.P., "Optimal Corrective Rescheduling for Power System Security", IEEE Trans. Power App. and Syst., Vol. PAS-90, No. 2, pp 843-851, March/April, 1971.
[8] Shen, C.M. and Laughton, M A., "Power System Load Scheduling with Security Constraints using Dual Linear programming', IEE Proceedings, Vol. 117, No. 11, pp. 21172127, Nov. 1970.

[9] O. Alsac and B. Stott, "Optimal load flow with steady state security," IEEE Trans. on Power Apparatus and Systems, Vol. PAS-93, pp. 745-751, May/June 1974

[10] Monticelli, A.; Pereira, M. V. F.; Granville, S., "SecurityConstrained Optimal Power Flow with Post-Contingency Corrective Rescheduling," Power Systems, IEEE Transactions on , vol.2, no.1, pp.175-180, Feb. 1987

[11] Carpentier, J., "Differential Injections Method: A General Method for Secure and Optimal Load Flows", IEEE PICA Conference Proceedings Minneapolis, MN, pp. 255-262, June 1973

[12] Elacqua, A. J. and Corey, S. L., "Security Constrained Dispatch at the New York Power Pool", IEEE Trans. Power App. Syst., Vol. PAS-101, pp. 2876-2884, Aug. 1982.

[13] Schnyder, G. and Glavitsch, H., "Integrated Security Control Using an Optimal Power Flow and Switching Concepts", IEEE PICA Conference Proceedings, Montreal, Canada, pp. 429436, May 1987

[14] Stott, B. and Hobson, E., "Power System Security Control Calculations Using Linear Programming Pam I and 2", IEEE Trans. Power App. Systems., Vol. PAS-97, pp. 1713-1731, Sept./Oct. 1978

[15] Dias, L.G. and El-Hawary, M.E., "Effects of Load Modeling in Security Constrained OPF Studies", IEEE Trans. on Power Systems, Vol. 6, No.2, pp. 87-93, February 1991

[16] Saavedra, O.R., "Solving the security constrained optimal power flow problem in a distributed computing environment," Generation, Transmission and Distribution, IEE Proceedings, vol.143, no.6, pp.593-598, Nov 1996

[17] Rodrigues, M.; Saavedra, O.R.; Monticelli, A., "Asynchronous programming model for the concurrent solution of the security constrained optimal power flow problem," Power Systems, IEEE Transactions on, vol.9, no.4, pp.2021-2027, Nov 1994

[18] F. Gagliardi, E Chiodo, D Mehnniti and A. Tesla, "A pattern recognition approach for steady state security 
evaluation of an Electrical Power system" European Trans on Electrical Power Engineering, Vol. I, No.5. pp. 243-251, 1991.

[19] D Menniti, D Picardi, C Sorrentino, N Tesla, "Neural network based prediction of the steady state security for electrical power system," 2nd IFAC Sipower'95, Mexico Mexico, 6-8 December 1995

[20] Conforti, D.; Menniti, D.; Sorrentino, N.; Verde, P.; Testa, A., "Optimal load-flow with $\mathrm{N}-1$ steady-state security via high performance computing," Electrotechnical Conference, 1996. MELECON '96., 8th Mediterranean , vol.2, no., pp.873-879 vol.2, 13-16 May 1996

[21] E.Vaahedi, Y.Mansour, C.Fuches, S. Granville, M.Latore, H.Hamadanizadeh, "Dynamic Security Constrained Optimal Power Flow/VAr Planning", IEEE Transaction on Power Systems, Vol. 16, No. 1, pp 38-43, February 2001

[22] Hur, D.; Jong-Keun Park; Kim, B.H.; Kwang-Myoung Son, "Security constrained optimal power flow for the evaluation of transmission capability on Korea electric power system," Power Engineering Society Summer Meeting, 2001. IEEE, vol.2, pp.1133-1138, 2001

[23] Lizhi Wang, Mainak Mazumdar, "Contingency Selection in Security Constrained Optimal Power Flow Problem: A Multi-Objective Approach", Electricity Conference 2006

[24] Y. Yuan, J. Kubokawa, and H. Sasaki, "A solution of optimal power flow with multi-contingency transient stability constraints," IEEE Trans. on Power Systems, Vol. 18, No. 3, pp. 1094-1102, Aug. 2003

[25] F. Bouffard, F. D. Galiana, J. M. Arroyo. "Umbrella Contingencies in Security-Constrained Optimal Power Flow". 15th Power Systems Computation Conference, PSCC'05. Liege, Belgium. August 2005

[26] Capitanescu, F.; Glavic, M.; Ernst, D.; Wehenkel, L., "Contingency Filtering Techniques for Preventive SecurityConstrained Optimal Power Flow," Power Systems, IEEE Transactions on , vol.22, no.4, pp.1690-1697, Nov. 2007

[27] V. C. Ramesh and Xuan Li, "A fuzzy multi-objective approach to contingency constrained OPF", IEEE Trans. Power Syst., vol. 12, no. 3, pp.1348-1354, Aug. 1997

[28] Devaraj, D.; Yegnanarayana, B., "Genetic-algorithm-based optimal power flow for security enhancement," Generation, Transmission and Distribution, IEE Proceedings- , vol.152, no.6, pp. 899-905, 4 Nov. 2005

[29] Lopez-Lezama, J.M.; Murillo-Sanchez, C.E.; Zuluaga, L.J.; Gutierrez-Gomez, J.F., "A Contingency-Based SecurityConstrained Optimal Power Flow Model For Revealing The Marginal Cost of a Blackout Risk-Equalizing Policy in the Colombian Electricity Market," Transmission \& Distribution Conference and Exposition: Latin America, 2006. TDC '06. IEEE/PES, vol., no., pp.1-6, 15-18 Aug. 2006

[30] Aganagic, M.; Mokhtari, S., "Security constrained economic dispatch using nonlinear Dantzig-Wolfe decomposition,"
Power Systems, IEEE Transactions on , vol.12, no.1, pp.105-112, Feb 1997

[31] Yan, X.; Quintana, V.H., "An efficient predictor-corrector interior point algorithm for security-constrained economic dispatch," Power Systems, IEEE Transactions on , vol.12, no.2, pp.803-810, May 1997

[32] Vargas, L.S.; Quintana, V.H.; Vannelli, A., "A tutorial description of an interior point method and its applications to security-constrained economic dispatch," Power Systems, IEEE Transactions on , vol.8, no.3, pp.1315-1324, Aug 1993

[33] Jabr, R.A.; Coonick, A.H.; Cory, B.J., "A homogeneous linear programming algorithm for the security constrained economic dispatch problem," IEEE Transactions on Power Systems, vol.15, no.3, pp.930-936, Aug 2000

[34] Yong Fu; Shahidehpour, M.; Zuyi Li, "AC contingency dispatch based on security-constrained unit commitment," Power Systems, IEEE Transactions on , Vol.21, No.2, pp. 897-908, May 2006

[35] Kyoung-Shin Kim; Leen-Hark Jung; Seung-Chul Lee; UnChul Moon, "Security Constrained Economic Dispatch Using Interior Point Method, "International Conference on Power System Technology, PowerCon 2006. pp.1-6, 22-26 Oct. 2006

[36] Capitanescu, F.; Wehenkel, L., "A New Iterative Approach to the Corrective Security-Constrained Optimal Power Flow Problem," Power Systems, IEEE Transactions on , vol.23, no.4, pp.1533-1541, Nov. 2008

[37] Harsan, H.; Hadjsaid, N.; Pruvot, P., "Cyclic security analysis for security constrained optimal power flow," IEEE Transactions on Power Systems, vol.12, no.2, pp.948-953, May 1997

[38] Zaoui, F.; Fliscounakis, S., "A Direct Approach for the Security Constrained," Power Systems Conference and Exposition, 2006. PSCE '06. 2006 IEEE PES , vol., no., pp.1562-1569, Oct. 29 2006-Nov. 1, 2006

[39] P. E. Onate Yumbla, J. M. Ramirez, and C. A. Coello, "Optimal power flow subject to security constraints solved with a Particle swarm optimizer," IEEE Transactions on Power Systems, Vol. 23, No. 1, pp. 33-40, February 2008

[40] L. L. Lai, J. T. Ma, R. Yokoyama, and M. Zhao, “ Improved genetic algorithms for optimal power flow under both normal and contingent operation states," Electrical Power \& Energy Systems, Vol. 19, No. 5, pp. 287-292, June 1997

[41] P.Somasundaram, K., Kuppusamy, and Kumudini Devi, "Evolutionary programming based security constrained optimal power flow", Electric Power System Research, Elsevier, pp. 137-145, 2004

[42] Zwe-Lee Gaing; Rung-Fang Chang, "Security-constrained optimal power flow by mixed-integer genetic algorithm with arithmetic operators," IEEE Power Engineering Society General Meeting, pp. 1-8, 2006 
[43] Stott, B.; Alsac, O.; Monticelli, A.J., "Security analysis and optimization," Proceedings of the IEEE, Vol.75, No.12, pp. 1623-1644, Dec. 1987

[44] J. A. Momoh and J. Zhu, "Multi-area power systems economic dispatch using nonlinear convex network flow programming", Electric Power Syst. Research, vol. 59, pp. 13-20, 2001

[45] Karoui, K.; Platbrood, L.; Crisciu, H.; Waltz, R.A., "New large-scale security constrained optimal power flow program using a new interior point algorithm," Electricity Market, 2008. EEM 2008. 5th International Conference on European , vol., no., pp.1-6, 28-30 May 2008

[46] Anibal T. de Azevedo, Carlos A. Castro, Aurelio R.L. Oliveira, Secundino Soares, "Security constrained optimal active power flow via Network model and interior point method", Revista Controle \& Automação/Vol.20 no.2, pp 206-216/Abril, Maio e Junho 2009

[47] Claudio Cañizares, William Rosehart, Alberto Berizzi, Cristian Bovo, "Comparison of Voltage Security Constrained Optimal Power Flow Techniques", Proc. 2001 IEEE-PES Summer Meeting, Vancouver, BC, July 2001

[48] D. Devaraj, J. Preetha Roselyn, "Improved genetic algorithm for voltage security constrained optimal power flow problem", International Journal of Energy Technology and Policy, Vol. 5, No. 4, pp.475-488, 2007

[49] Nakawiro, W.; Erlich, I., "A Combined GA-ANN Strategy for Solving Optimal Power Flow with Voltage Security Constraint," Power and Energy Engineering Conference, 2009. APPEEC 2009. Asia-Pacific, vol., no., pp.1-4, 27-31 March 2009

[50] Biskas, P.N.; Bakirtzis, A.G., "A decentralized solution to the Security Constrained DC-OPF problem of multi-area power systems," Power Tech, 2005 IEEE Russia , pp.1-7, pp. 27-30, June 2005

[51] Don Hur, Kim Balho, "Application of Distributed Optimal Power Flow to Power System Security Assessment", Journal of Electric Power Components and Systems, Taylor \& Francis, Vol. 31, Issue. 1, pp. 71-80, 2003

[52] Wei Qiu; Flueck, A.J.; Feng Tu, "A new parallel algorithm for security constrained optimal power flow with a nonlinear interior point method," Power Engineering Society General Meeting, 2005. IEEE, pp. 447-453 Vol. 1, 12-16 June 2005

[53] Borges, C.L.T.; Alves, J.M.T., "Power System Real Time Operation based on Security Constrained Optimal Power Flow and Distributed Processing," Power Tech, 2007 IEEE Lausanne, vol., no., pp.960-965, 1-5 July 2007
[54] C. Lehmköster, "Security constrained optimal power flow for an economical operation of FACTS-devices in liberalized energy markets," IEEE Trans. Power Del., vol. 17, no. 2, pp. 603-608, Apr. 2002

[55] Berizzi, A.; Delfanti, M.; Marannino, P.; Pasquadibisceglie, M.S.; Silvestri, A., "Enhanced Security-Constrained OPF With FACTS Devices," Power Systems, IEEE Transactions on , vol.20, no.3, pp. 1597-1605, Aug. 2005

[56] Glanzmann, G.; Andersson, G., "Incorporation of N-1 Security into Optimal Power Flow for FACTS Control," Power Systems Conference and Exposition, 2006. PSCE '06. 2006 IEEE PES, vol., no., pp.683-688, Oct. 29 2006-Nov. 1,2006

[57] R, Narmatha Banu, D, Devaraj, "Enhanced genetic algorithm approach for Security constrained optimal power flow Including FACTS devices", International Journal of Electrical Power and Energy Systems Engineering, pp. 3338,2009

[58] T.S. Prasanna, N.B. Muthuselvan, P. Somasundaram, "Security constrained OPF by Fuzzy stochastic Algorithms in Interconnected Power Systems", Journal of Electrical Engineering, 5-1, 2009

[59] G. Hug-Glanzmann, G. Andersson, "N-1 security in optimal power flow control applied to limited areas", IET Generation, Transmission and Distribution, Vol. 3, Issue. 2, pp. 206-215, 2009

[60] F. Capitanescu, M. Glavic, D. Ernst, L. Wehenkel, "Applications of Security-Constrained Optimal Power Flows", Modern Electric Power Systems Symposium, MEPS06, Wroclaw, Poland, September 2006

[61] R Jayashree, M Abdullah Khan, "A Mutli-Transaction based Security Constrained Optimal Power Flow tool for Assessment of Available transfer capability and Congestion Management", International Journal of Electrical and Power Engineering, pp. 501-511, 2007

[62] Sarbjeet Kaur Bath, Jaspreet Singh Dhillon, and D P Kothari. "Security Constrained Stochastic Multi-objective Optimal Power Dispatch" International Journal of Emerging Electric Power Systems 8.1, 2007

[63] Thomas, R.J.; Murillo-Sanchez, C.E.; Zimmerman, R.D., "An advanced security constrained OPF that produces correct market-based pricing," Power and Energy Society General Meeting - Conversion and Delivery of Electrical Energy in the 21st Century, 2008 IEEE , vol., no., pp.1-6, 20-24 July 2008 